Supporting Information for

\title{
Tracking Longitudinal Rotation of Silicon Nanowires for Biointerfaces
}

Youjin V. Lee, ${ }^{\dagger}$ David Wu, ${ }^{*} *$ Yun Fang, ${ }^{*}$ Yuxing Peng,,$*$ and Bozhi Tian ${ }^{\dagger, 1 * *}$

${ }^{\dagger}$ Department of Chemistry, University of Chicago, Chicago, 60637

Department of Medicine, Section of Pulmonary and Critical Care Medicine, University of Chicago, Chicago, 60637

"Research Computing Center, University of Chicago, Chicago, 60637

$\perp$ James Franck Institute, The University of Chicago, Chicago, 60637

*Corresponding authors: Bozhi Tian (btian@uchicago.edu), David Wu (dwu1@medicine.bsd.uchicago.edu), Yuxing Peng (yuxing@uchicago.edu) 


\section{Methods and Materials}

Kinked-nanowires synthesis. Kinked silicon nanowires were synthesized via the vaporliquid-solid mechanism, using gold nanocrystals as catalysts. Au nanoparticles with $50 \mathrm{~nm}$ diameters (TedPella, diluted 9x with $10 \%$ HF solution) were deposited onto a $\mathrm{Si}<100>$ substrate (Nova Electronic Materials, n-type). The nanowire growth was carried out at $480{ }^{\circ} \mathrm{C}$, and 40 torr in a home-built chemical vapor deposition system. Silane $\left(\mathrm{SiH}_{4}\right)$ was used as the silicon source, hydrogen $\left(\mathrm{H}_{2}\right)$ as a carrier gas, and phosphine $\left(\mathrm{PH}_{3}, 1000 \mathrm{ppm}\right.$ in $\left.\mathrm{H}_{2}\right)$ as the n-dopant source. $\mathrm{SiH}_{4}, \mathrm{H}_{2}$, and $\mathrm{PH}_{3}$ were flown in at the rate of 60,2 , and 2 standard cubic centimeters per minute, respectively. To introduce a kink, the system was purged toward the based pressure of about 1 mTorr in between the growth of the arm and the main body part. The growth order was as follows: arm growth for $45 \mathrm{~s}$, system purge for $15 \mathrm{~s}$, main body growth for $25 \mathrm{~min}$, system purge for $15 \mathrm{~s}$, and arm growth for $45 \mathrm{~s}$. This synthesis resulted in nanowires with one or two kinks at each ends. Note, not all purge steps successfully introduced a kink. Nanowires used in the experiments had only one kink because nanowires often break at some part of the main body upon sonication, leaving only one kink per NW. Resulting nanowires were polydisperse: their diameters ranged from $10 \mathrm{~nm}$ to $200 \mathrm{~nm}$ (SI Appendix, Fig. S5).

Cell culture. Human umbilical vein endothelial cells (HUVEC) were cultured using M200 growth media (Life Technologies) with Low Serum Growth Supplement (Thermo Fisher) and Antibiotic-Antimycotic (Thermo Fisher). Cell cultures were stored at $37^{\circ} \mathrm{C}$ and under $5 \% \mathrm{CO}_{2}$ in a sterile incubator. Cells at passage seven were used for the experiment.

Experiment setup. Because 3D motions of a nanowire complicate the tracking, we settled the nanowires near the substrate bottom or placed the nanowires in contact with a cell that was lying flat; this suppressed any significant out-of-plane rotations. We confirmed that the nanowire 
remained in pseudo 2D space by measuring its body length: if the nanowire remains in the twodimensional space, the projected length of the nanowire's main body part should be constant (S10).

i. Non-interacting case. The nanowire floated in media on top of the extracellular matrix: Nanowire-M200 media mixture was freshly prepared by sonicating the SiNWs into the media. After the original media was aspirated, the prepared nanowire-media mixture $(\sim 400 \mu \mathrm{L})$ was added to the cell culture in a $35 \mathrm{~mm}$ plastic Petri dish. The dish was placed in a preheated microincubator installed on the microscope. Videos of the nanowires were taken within the first five hours after the nanowire-media mixture was added. Due to the considerable time ( $>3 \mathrm{hr})$ it takes for the nanowires to settle to the bottom of the dish, no observed nanowires were in contact with the cells on the bottom of the dish.

ii. Cell-interacting case. The nanowire was in contact with a HUVEC. Twenty-four hours prior to the experiment, the nanowire-media mixture $(\sim 400 \mu \mathrm{L})$ was added to an empty $35 \mathrm{~mm}$ Petri dish. The dish was stored in the $37^{\circ} \mathrm{C}$ sterile incubator overnight to allow the nanowires to settle at the bottom of the dish. On the day of the experiment, HUVEC were thawed and resuspended in the M200 media (1.4 mL). After aspirating media in the dish, leaving the nanowires behind, we seeded the HUVEC. We placed the dish inside a microincubator and started acquiring data immediately. Seeding the cells on top of the settled nanowires, as opposed to the other way around, ensures interaction between nanowire and cell, as the heavy cells settle down much faster than the nanowires.

Data acquisition. Videos of the nanowire motions were acquired using an IX71 inverted microscope (Olympus) and ORCA-R2 CCD (Hamamatsu) detector. During the data acquisition, the magnification of the objective lens was matched to pixel size such that the nanowire covered 
at least five pixels in the longitudinal dimension, sufficient for Nyquist sampling ${ }^{1}$. Videos were acquired in two channels. We recorded the first channel in the scatter-enhanced phase contrast (SEPC) mode for visualizing both the cells and the nanowires ${ }^{2}$. In the SEPC mode, we used both the internal lamp and the LED-144-YK ring LED lamp (Amscope) mounted around the condenser. We recorded the second channel in the scatter-enhanced pseudo darkfield (SEPD) mode for visualizing only the nanowires. Here, only the ring LED lamp was turned on; turning off the internal lamp, thus minimizing signal from the cells, facilitated the nanowire detection in the data processing stage. During data acquisition, the sample dishes were kept inside an INUBONICS-F1 stage top microincubator (Tokai Hit, $37{ }^{\circ} \mathrm{C}, 5 \% \mathrm{CO} 2$ ).

Image processing. Application of the Gaussian centerline detection algorithm significantly improves the accuracy of the PCA-based arm detection in the next step. Due to their high scattering efficiency, silicon nanowires appear more than ten times thicker than their actual diameter under the optical microscope ${ }^{3}$. This apparent radius or thickness can result in inaccurate measurement of $\mathrm{PL}_{\mathrm{arm}}$, defined as the distance between the arm end and the main body center (Figure S2). The Gaussian centerline detection algorithm also addresses other problems presented by the non-zero apparent thickness of the nanowire. First, when solving Eq. 1 for longitudinal orientation $(\theta)$ in the following step, the $r \sin \theta$ term in Eq. 1 causes ambiguity in $P L_{\text {arm }}$ near $0^{\circ}$ and $180^{\circ}$ (Figure S3). Second, when the nanowire is near $90^{\circ}$, the arm aligns with the main body and becomes shadowed. By narrowing the nanowire selection (i.e. making the apparent radius of the nanowire $(r)$ almost negligible), the Gaussian centerline detection algorithm greatly reduces the ambiguous regions near $0^{\circ}$ and $180^{\circ}$. As a result, we utilized $80 \%$ of the non-interacting video and $98 \%$ of the cell-interacting video (Table S1). Note that more 
frames were discarded for the non-interacting video as its nanowire rolled more vigorously and more frequently crossed the shadowed region.

Calculation (Converting PL $_{\text {arm }}$ to longitudinal rotation). We solved Eq. 1 in the main text to convert $P L_{\text {arm }}$ to longitudinal orientation. Based on the longitudinal orientations, we calculated longitudinal rotation. We defined the nanowire longitudinal orientation to range between $0^{\circ}$ and $180^{\circ}$ because we cannot distinguish whether the nanowire arm is pointing towards or away from the detector. To track rolling motion, we are interested in the relative orientations of the nanowire; therefore, our approach is not affected by an absence of absolute orientation measurements (Supporting information S6).

In order to prevent underestimation due to confining the $\theta(t)$ to span between $0^{\circ}$ and $180^{\circ}$, we fit the $\mathrm{MSAD}_{\text {longitudinal }}$ up to $\mathrm{t}=0.5 \mathrm{~s}$ where the plot increases monotonically, meaning the observed time period is shorter than the relaxation time. Depending on the study in question, different ways of fitting the parameters can be considered, as discussed by $\mathrm{Wu}$ and Berland ${ }^{4,5}$. A particle's rotations can deviate from normal diffusion $(\alpha=1)$ to either super-diffusion $(\alpha>1)$ or sub-diffusion $(\alpha<1)$. In intracellular settings, these anomalous diffusions are often observed. For example, particles like organelles and vesicles undergo super-diffusive translations when they are actively transported by molecular motors ${ }^{6}$, actin, and microtubules in cytoplasm ${ }^{7,8}$. Particles also undergo sub-diffusive translations in a crowded environment or when they interact with stationary organelles 9,10 . Less attention has been paid to rotational motion compared to translational motion due to technical limitations of measuring rotation, but we expect to see nonnormal diffusive behavior with rotation. While diffusivity coefficient (D) roughly corresponds to the instantaneous speed of the rotation, a direct comparison of the diffusivity coefficient (D) is 
less meaningful than a direct comparison of the anomalous exponent due to the varying unit $\left(\operatorname{deg}^{\alpha} / s\right)$, while the alpha parameter is unit-less.

Error Analysis. Based on the analysis of immobilized nanowires, we measured the 95\% confidence interval of the projected length of the arm to be \pm 0.66 pixels, which corresponds to \pm $67 \mathrm{~nm}$. This tracking inaccuracy translates to inaccuracy in longitudinal orientation according to the Eq. 1 in the main text. Next, we calculate the fitting errors associated with the anomalous exponents and the diffusivity coefficients were insignificant, as shown in the Figure 3C, and Figure $4 \mathrm{C}$ in the main text.

\section{References}

(1) Crocker, J. C.; Grier, D. G. Methods of Digital Video Microscopy for Colloidal Studies. J. Colloid Interf. Sci. 1996, 179 (1), 298-310.

(2) Zimmerman, J. F.; Murray, G. F.; Wang, Y.; Jumper, J. M.; Austin, J. R.; Tian, B. FreeStanding Kinked Silicon Nanowires for Probing Inter- and Intracellular Force Dynamics. Nano Lett. 2015, 15 (8), 5492-5498.

(3) Brönstrup, G.; Jahr, N.; Leiterer, C.; Csáki, A.; Fritzsche, W.; Christiansen, S. Optical Properties of Individual Silicon Nanowires for Photonic Devices. ACS Nano 2010, 4 (12), 7113-7122.

(4) Wu, J.; Berland, K. M. Propagators and Time-Dependent Diffusion Coefficients for Anomalous Diffusion. Biophys. J. 2008, 95 (4), 2049-2052.

(5) Kepten, E.; Weron, A.; Sikora, G.; Burnecki, K.; Garini, Y. Guidelines for the Fitting of Anomalous Diffusion Mean Square Displacement Graphs from Single Particle Tracking Experiments. PLoS One 2015, 10 (2).

(6) Mallik, R.; Gross, S. P. Molecular Motors: Strategies to Get Along. Curr. Biol.2004, 14 (22), R971-R982.

(7) Reverey, J. F.; Jeon, J.-H.; Bao, H.; Leippe, M.; Metzler, R.; Selhuber-Unkel, C. Superdiffusion Dominates Intracellular Particle Motion in the Supercrowded Cytoplasm of Pathogenic Acanthamoeba Castellanii. Sci. Rep. 2015, 5.

(8) Bruno, L.; Levi, V.; Brunstein, M.; Despósito, M. A. Transition to Superdiffusive Behavior in Intracellular Actin-Based Transport Mediated by Molecular Motors. Phys. Rev. E 2009, 80 (1), 011912.

(9) Otten, M.; Nandi, A.; Arcizet, D.; Gorelashvili, M.; Lindner, B.; Heinrich, D. Local Motion Analysis Reveals Impact of the Dynamic Cytoskeleton on Intracellular Subdiffusion. Biophysical J. 2012, 102 (4), 758-767.

(10) Katz, Z. B.; English, B. P.; Lionnet, T.; Yoon, Y. J.; Monnier, N.; Ovryn, B.; Bathe, M.; Singer, R. H. Mapping Translation "hot-Spots" in Live Cells by Tracking Single Molecules of MRNA and Ribosomes. eLife 2016, 5, e10415. 


\section{S1. Rotational inertias associated with different types of rotations}

Torque associated with a polar rotation of mass $M$, is a function of the object's length $(l)$ squared. On the other hand, that of a rolling motion is a function of the object's radius $(R)$ squared.

Rotational inertias for a thin rod about center:

$$
I=\frac{1}{12} M l^{2}
$$

Rotational inertias for a thing rod about end:

$$
I=\frac{1}{3} M l^{2}
$$

Rotational inertias for a solid cylinder about its axis:

$$
I=\frac{1}{2} M R^{2}
$$

In case of a nanowire with 1:100 radius to length aspect ratio (similar to the nanowires used in the reported experiments), the torque of rolling motion is more three orders of magnitude smaller than that of in-plane rotation. 


\section{S2. Determination of the arm length and the nanowire thickness}

As shown in Eq. 1 in the main text, the arm length (1) and the nanowire thickness (r) are the two measured parameters involved in the determination of the nanowire's longitudinal orientation based on the projected length of arm. For a consistent data processing, we determine the two parameters in a systematic and logical ways.

Determination of the nanowire radius (r)

Although the term radius is used, the measured radii are likely to be larger than the actual radii of the nanowire due to the strong scattering of Si. Nanowire radius is determined by taking the average of the absolute values of the measured radius of every frame in a video. In each frame, radius is measured to be the maximum distance between the Gaussian centerline coordinates near the main-body end and the principal component. For consistency when processing multiple videos of the same nanowire, the minimum value of the nanowire radii from the videos is used. We used the minimum value, because the measured value is likely to be an overestimation of the true radius.

Determination of the arm length (1)

$$
l=\sqrt{P L_{a r m, \max }^{2}-r^{2}}
$$

First, top $1 \%$ of the largest absolute values of $P L_{a r m}$ are removed from the list of $P L_{a r m}$. The removal of the largest values ensures removal of falsely largely detected $P L_{\text {arm }}$. Finally, the largest of the remaining $P L_{a r m}$ is defined to be the $P L_{a r m, \max }$. When more than one video of the same sample was taken, the largest of $P L_{a r m, \max }$ determined from each video was used as the $P L_{a r m, \max }$ for all the videos. Also, we make sure that the nanowires are oriented near their parallel orientations (near $0^{\circ}$ or $180^{\circ}$ ) at least once in the observed time. 

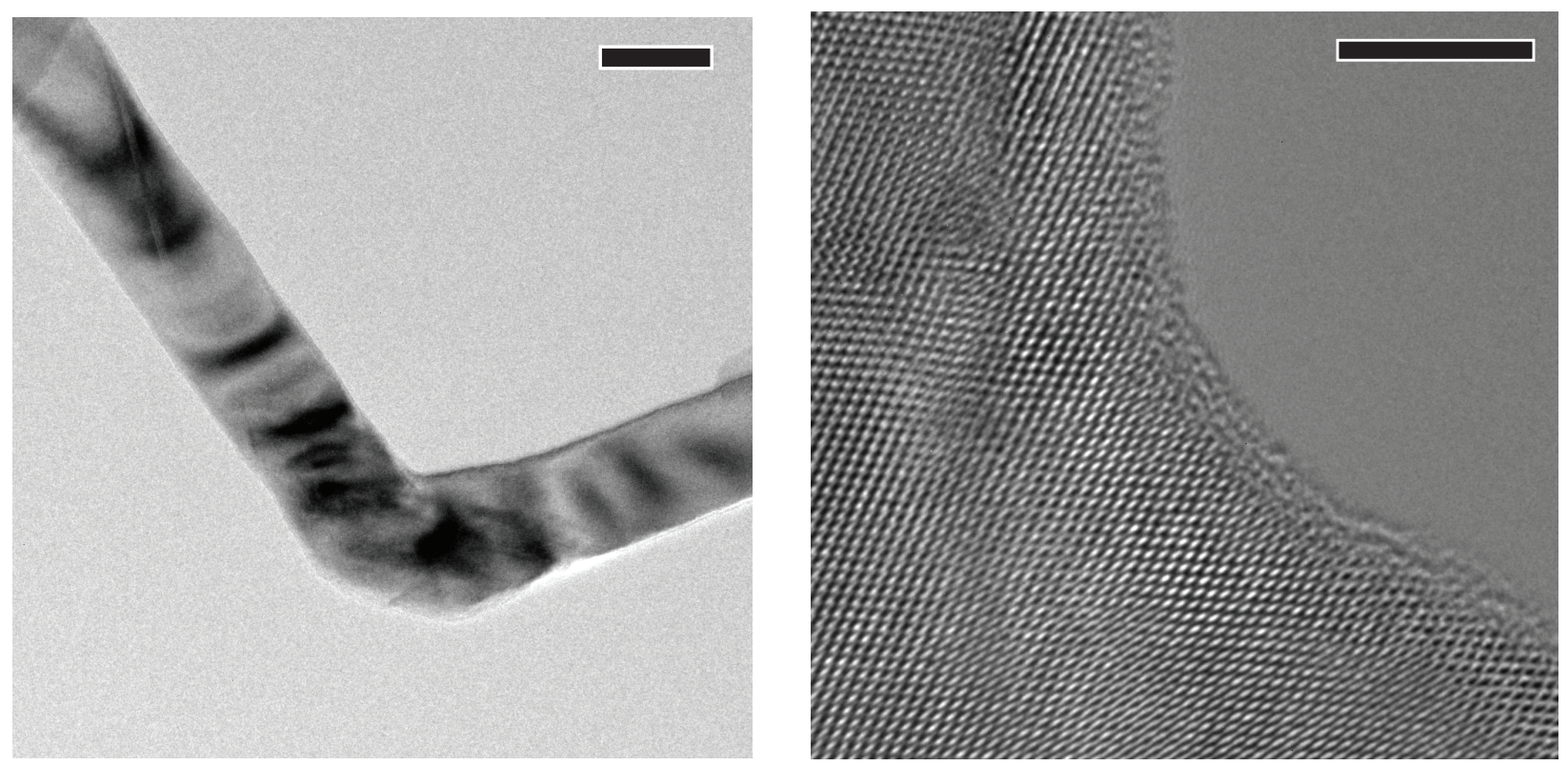

Figure S1. Transmission electron microscopy images. Transmission electron microscopy images of the kinked portion of the kinked silicon nanowire. Left scale bar: $50 \mathrm{~nm}$, right scale bar: $5 \mathrm{~nm}$. 


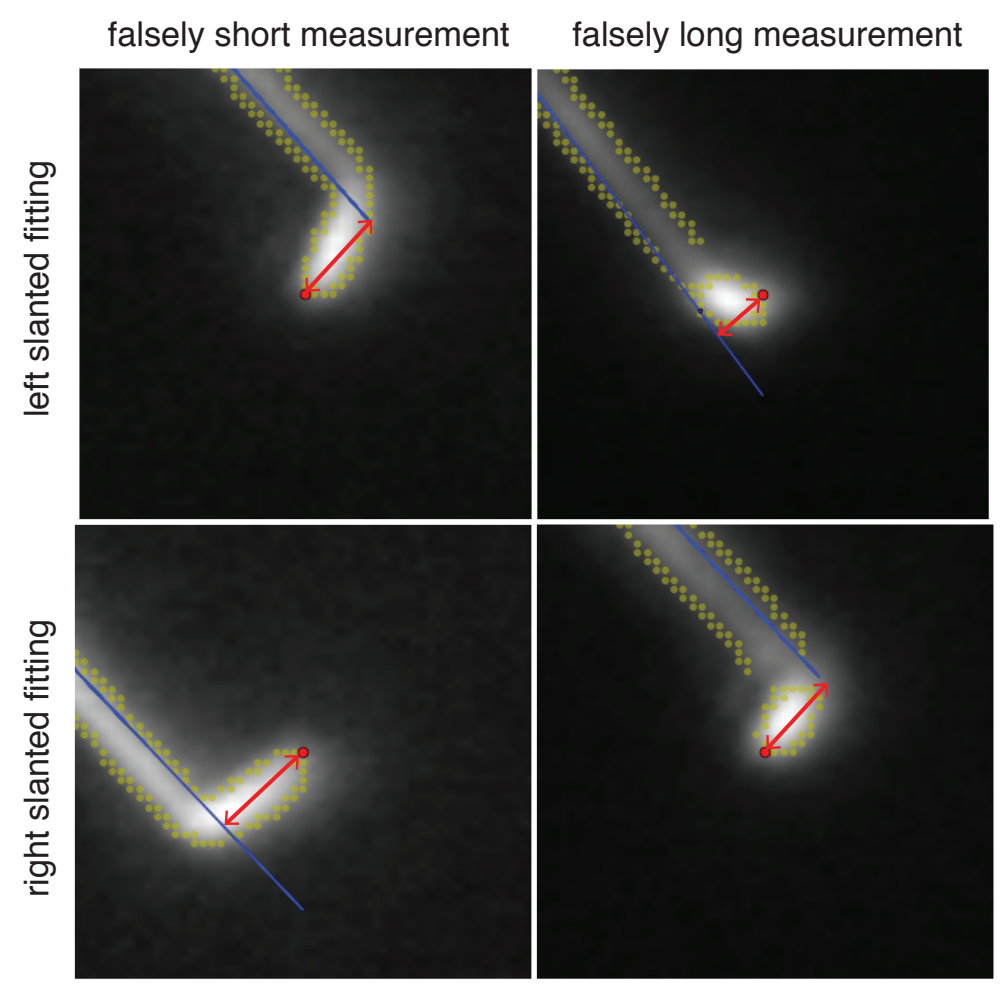

Figure S2. Inaccurate detection of $\mathrm{PL}_{\text {arm }}$ when not applying the Gaussian centerline detection algorithm. Yellow dots are the threshold selected outline of the nanowire. Blue solid line is the fitted center of the nanowire main-body part. The red doubled sided arrow indicates the measure $\mathrm{PL}_{\text {arm }}$. 

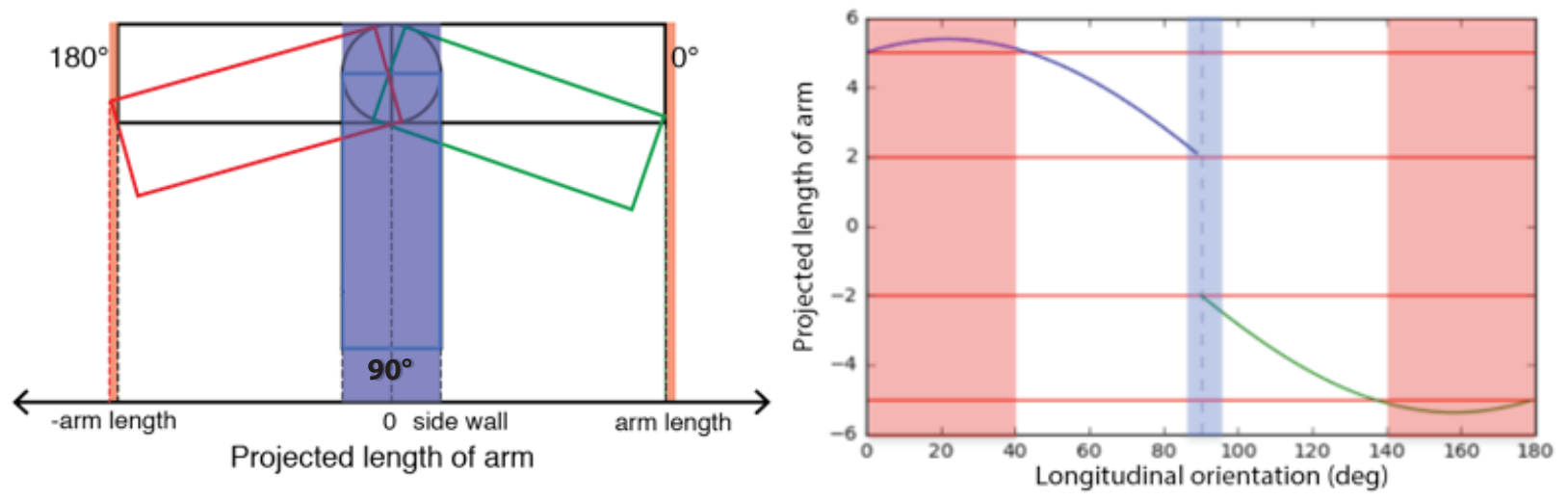

Figure S3. Left: schematics of the nanowire arm in various orientations and their corresponding $\mathrm{PL}_{\text {arm. }}$. Right: plot of the $\mathrm{PL}_{\text {arm }}$ 's for every possible longitudinal orientations. Here, the apparent thickness of the nanowire (r) is 2 and the arm length is 4 . Both: red regions are the ambiguous range, blue regions are the shadowed range. 


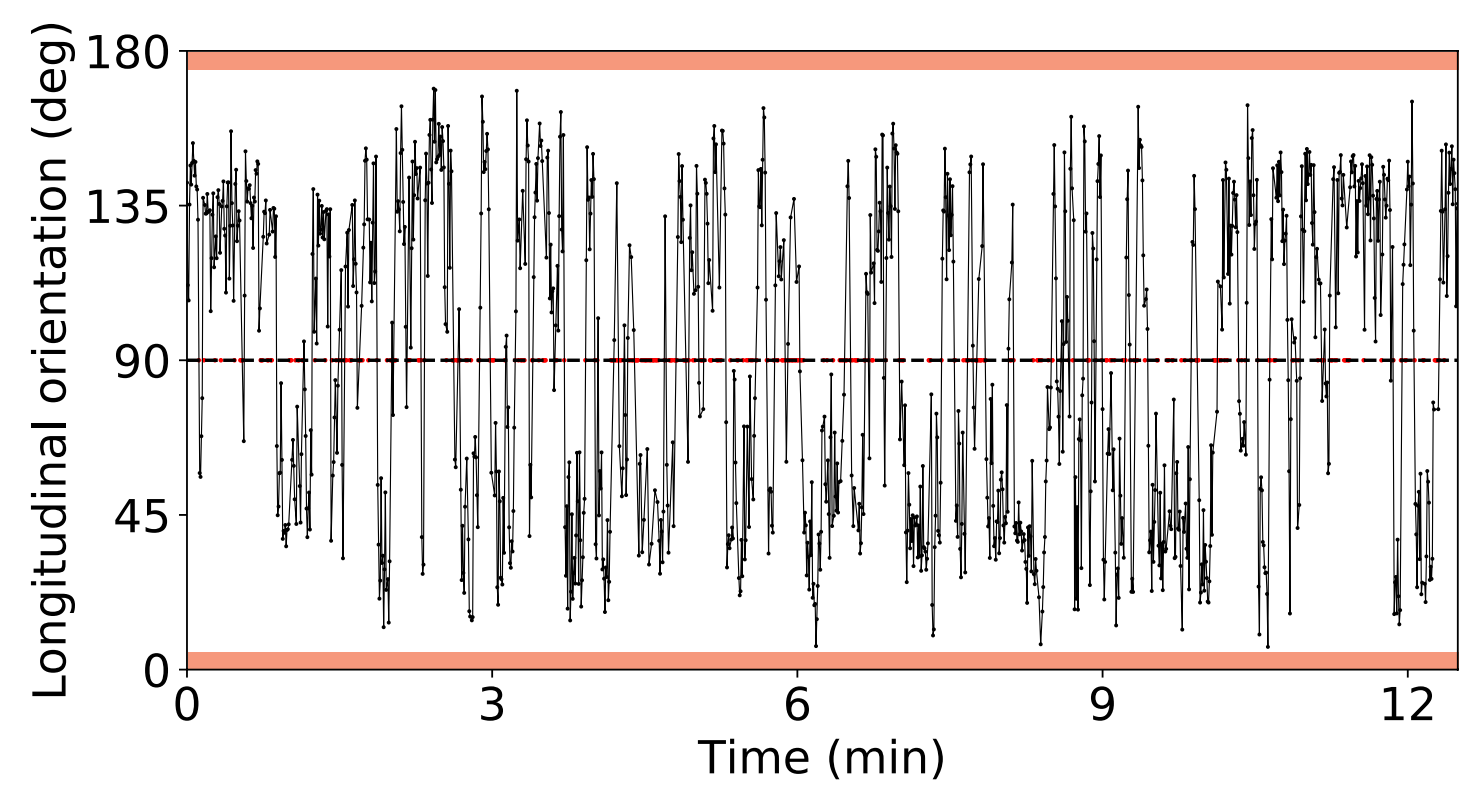

Figure S4. Longitudinal orientation trajectory of a free-floating nanowire. The longitudinal orientation vs. time plot of the entire free-floating nanowire experiment. The red dots along the $90^{\circ}$ horizontal line indicate the indices of removed frames due to either being ambiguous (when the nanowire is oriented near $0^{\circ}$ or $180^{\circ}$ ) or being shadowed by the nanowire main body (when the nanowire is oriented near $90^{\circ}$ ). The red regions near $0^{\circ}$ and $180^{\circ}$ indicate the range where data points are removed to prevent under-estimation of the longitudinal rotation. For example, if the nanowire rotates from $358^{\circ}$ to $3^{\circ}$ (5 degrees of rotation), it will appear to have rotated from $2^{\circ}$ to $3^{\circ}$ ( 1 degree of rotation). Of note, these frames are already discarded as they also fall in the ambiguous region. 

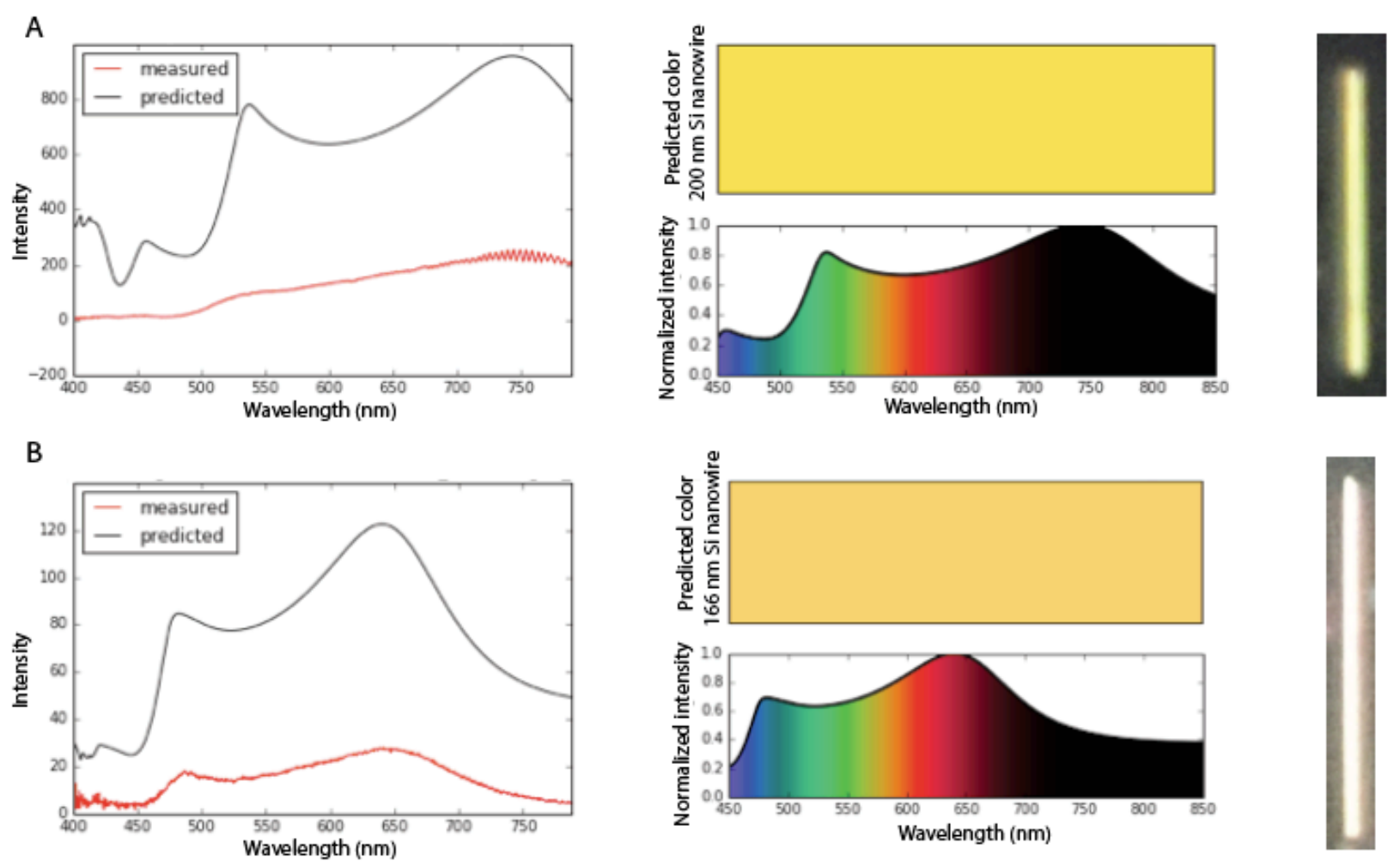

Figure S5. Nanowire thickness measurement. Using the Fergie spectroscopy (Princeton Instrument), attached to the same microscope setup, we measured the nanowire thickness based on the analysis illustrated in ref. 17 in the main text. Thickness of the probe can be measured concurrently with the video acquisition. Two sets of data are presented in panel A and B. In each panel, right is the measured spectrum and the closest matching calculated spectrum plotted over wavelength. In the middle are the calculated spectrum (bottom) and the color based on spectrum (top). On the right is a colored photography (iPhone6) taken through the microscope's eyepiece that matches the predicted color. 
Table S1. \% utilization of the data

\begin{tabular}{|c|c|c|c|c|}
\hline Experiment & $\begin{array}{c}\text { Original \# of } \\
\text { frames }\end{array}$ & $\begin{array}{c}\text { \# of shadowed } \\
\text { frames }\end{array}$ & $\begin{array}{c}\text { \# of ambiguous } \\
\text { frames }\end{array}$ & $\begin{array}{c}\text { Utilization rate for data } \\
\text { processing (\%) }\end{array}$ \\
\hline Free-floating & 1500 & 14 & 16 & 77 \\
\hline Cell-interacting & 4500 & 80 & 5 & 98 \\
\hline
\end{tabular}


Table S2. Pseudo two-dimensional confinements.

\begin{tabular}{|c|c|c|c|}
\hline experiment & $\begin{array}{c}\text { MB average } \\
(\mu \mathrm{m})\end{array}$ & $\begin{array}{c}\text { MB SD } \\
(\mu \mathrm{m})\end{array}$ & $\begin{array}{c}\text { maximum observed } \\
\text { out-of-plane rotation }(\mathrm{deg})\end{array}$ \\
\hline Free-floating & 34.91 & 0.15 & 7.50 \\
\hline Cell-interacting_A & 12.039 & 0.096 & 10.21 \\
\hline Cell-interacting_B & 11.991 & 0.065 & 8.42 \\
\hline Cell-interacting_C & 11.958 & 0.144 & 12.52 \\
\hline
\end{tabular}

In order to determine whether the nanowire stays in the pseudo two-dimensional space, we kept track of the projected length of the nanowires' main-body part (MB). Based on the negligible fluctuations in the length, we concluded that the two nanowires underwent minimal out-of-plane rotations, and stayed in pseudo two-dimensional spaces. For the free-floating experiment, the projected length of the nanowire's main-body was $34.91 \pm 0.15 \mu \mathrm{m}$. First, we assumed the actual MB length to be the average MB length plus the standard deviation: $35.06 \mu \mathrm{m}$. Then, to estimate the maximum observed out-of-plane, we considered the minimum observed main-body part length, which is the average MB length minus the standard deviation: $34.76 \mu \mathrm{m}$. Based on these assumptions, we conclude the maximum out-of=plane rotation to be 7.5 degrees for the free-floating experiment.

Similarly, the relevant observed MB lengths and the calculated out-of-plane rotations of each experiment are reported in the table. Note that these out-of-plane are over-estimation of out-ofplane rotations as the standard deviation of the main-body lengths are within the detection fluctuations of the experiment. For example, the average main-body length varies more than $0.1 \mu \mathrm{m}$ among the cell-interacting cases, which uses the same nanowire. The individual mainbody length standard deviations are less than $0.1 \mu m$. 
Table S3. Fitting errors

\begin{tabular}{|c|c|c|}
\hline Experiment & Anomalous exponent $(\alpha)$ & Diffusivity coefficient (D). \\
\hline Free-floating & $0.079(13 \%)$ & $66(13 \%)$ \\
\hline Cell-interacting & $0.061(11 \%)$ & $0.90(14 \%)$ \\
\hline
\end{tabular}

Values in the table are $95 \%$ confidence intervals (relative to the average values). The errors in

fitting the $\mathrm{MSAD}_{\text {longitudinal }}$ to $D \Delta t^{\alpha}$ were calculated for the two fitting parameters, anomalous exponent $(\alpha)$ and the diffusivity coefficient (D). We used the estimated covariance given from scipy.optimize.curve_fit function in Python to calculate the standard deviation errors, which is the square root of the covariance. We are reporting the two parameters' errors only when their anomalous exponent was greater than 0.2 . For a non-rolling nanowire, characterized by the anomalous exponent of less than 0.2 , our model $\left(\mathrm{MSAD}_{\text {longitudinal }}=D \Delta t^{\alpha}\right)$ is inadequate because the $\mathrm{MSAD}_{\text {longitudinal }}$ may not be monotonically increasing. 
Video S1. MovieS1_free floating_50xRT.mov

Video of the free floating silicon nanowire taken in the bright field phase mode. The playtime is $50 \mathrm{x}$ the real time. The scale can be referred by the nanowire main body length, which is $35 \mu \mathrm{m}$.

Video S2. MovieS2_cell interacting_50xRT.avi

Video of a cell interacting silicon nanowire taken in the bright field phase mode. This video corresponds to the 0 to $12 \mathrm{~min}$ of the cell interacting data presented. The playtime is $50 \mathrm{x}$ the real time. The scale can be referred by the nanowire main body length, which is $12 \mu \mathrm{m}$.

Video S3. MovieS3_cell interacting_50xRT.avi

Video of a cell interacting silicon nanowire taken in the bright field phase mode. This video corresponds to the 30 to $42 \mathrm{~min}$ of the cell interacting data presented. The playtime is $50 \mathrm{x}$ the real time. The scale can be referred by the nanowire main body length, which is $12 \mu \mathrm{m}$.

Video S4. MovieS4_cell interacting_50xRT.avi

Video of a cell interacting silicon nanowire taken in the bright field phase mode. This video corresponds to the 110 to $112 \mathrm{~min}$ of the cell interacting data presented. The playtime is $50 \mathrm{x}$ the real time. The scale can be referred by the nanowire main body length, which is $12 \mu \mathrm{m}$.

Note: All videos are cropped and their resolutions were compromised in the process of converting PNG files to JPEG files. 